\title{
EDUCACIÓN Y CULTURA DE PAZ PARA EL CARIBE COLOMBIANO*
}

Brenda M. Valero Díaz

* Este artículo se deriva de la tesis doctoral "Género y paz: relatos de mujeres víctimas de violencia en Barranquilla-Colombia" adscrito al grupo de investigación "Derechos Humanos, Cultura de Paz, Conflictos y Postconflicto" de la Universidad Simón Bolívar de Barranquilla.

† Abogada egresada de la Universidad Libre, Especialista en Derecho de Familia con la Universidad Libre, Magíster en Educación de la Universidad Simón Bolívar, Candidata a Doctora sobre Estudios de la Mujeres, Discursos y Prácticas de Género de la Universidad de Granada (España), Docente universitaria e investigadora del grupo de investigación "Derechos Humanos, Cultura de Paz, Conflictos, Postconflicto", adscrito al Centro de Ciencias Sociales Humanas y Jurídica 
En este texto se exponen algunas ideas relativas a la incorporación de la cultura de paz, sus principios y valores en la práctica cotidiana, como fundamento de una educación que propicie la construcción de ciudadanía. Desde este marco surge la necesidad de desarrollar una cultura de paz con cobertura regional, que incorpore sus componentes básicos a la vida diaria como elemento transversal que favorezca todas aquellas acciones que conduzcan a la gestión pacífica de los conflictos.

Para que se alcance este propósito es importante que se implementen centros educativos libres de violencias, y para su consecución se hace inevitable que se examine de forma detallada el proceso de enseñanza-aprendizaje en niñas, niños, jóvenes, docentes y la comunidad en general ya que es trascendental que la cultura de paz nazca y se propicie dentro del aula como ejercicio que le apueste a la convivencia pacífica.

\section{¿QUÉ ES LA CULTURA DE PAZ?}

La Asamblea General de las Naciones Unidas (1999) define la cultura de paz como el conjunto de valores, actitudes, tradiciones, comportamientos y estilos de vida basados en el respeto a la vida, el fin de la violencia y la promoción y la práctica de la no violencia por medio de la educación, el diálogo y la cooperación; el respeto pleno a los principios de soberanía e independencia política; el respeto pleno y promoción de los derechos humanos y libertades fundamentales; el compromiso con el arreglo pacífico de los conflictos; la protección al medioambiente; el respeto a la igualdad y el fomento de los derechos de hombres y mujeres; derechos de todos a la libertad de expresión, opinión e información; adhesión a los principios de libertad, justicia, democracia, tolerancia, solidaridad, cooperación, pluralismo, diversidad cultural, 
diálogo y entendimiento entre todos los niveles de la sociedad entre las naciones.

Clive Harber (1997) expresa que la cultura de paz debe existir en los centros educativos como una propuesta que en definitiva favorezca en su alumnado experiencias democráticas, para que así puedan desarrollar en el aula y en la escuela las competencias, los valores y los comportamientos democráticos que contribuirán a la instauración de una cultura de paz y de la no-violencia.

Sin lugar a dudas es también muy importante que esta se encuentre relacionada con el desarrollo de una cultura de la democracia, ya que los gobiernos democráticos, al asegurar una paz duradera, se constituyen en un elemento fundamental sin el cual la cultura de paz sería imposible de alcanzar. Al respecto, Morachimo y Piscoya (2004, p.12) indican que "Las personas que actúan moralmente asumen su responsabilidad con los otros a través de una práctica sociopolítica consciente, que asegura una convivencia democrática y armónica". Por lo tanto, se podría agregar que la cultura de paz encierra también el compromiso, la voluntad y el deseo extensivo del individuo de querer modificar y transformar su contexto con sus actitudes, valores y comportamientos; estos le permitirán enfrentar los conflictos de forma pacífica y creativa.

\section{¿QUÉ ES EDUCAR PARA LA PAZ?}

Viçen Fisas (2001, p.1) sostiene:

"Educar para una cultura de paz significa educar para la crítica y la responsabilidad, para el conocimiento y el manejo positivo de los conflictos, así como para potenciar los valores del diálogo y el intercambio y revalorizar la práctica 
del cuidado y de la ternura. Todo ello como una educación pro-social que ayude a superar las dinámicas destructivas y afrontar las injusticias. Desde la educación para la paz se ha dicho siempre, y con razón, que hemos de educar para la disidencia, la indignación, la desobediencia responsable, la elección con conocimiento y la crítica, es decir, para salirnos de las propuestas de alienación cultural y política.

Por otro lado, Rodríguez Rojo (1994) pone de manifiesto la necesidad de analizar el mundo en que vivimos, sometiéndolo a la crítica reflexiva que emana de los valores del pacifismo, para promover en las personas un compromiso transformador que, afianzado en la búsqueda de la verdad, pueda contribuir a la emancipación de todos y de sí mismas en primer lugar. En este sentido se posiciona también Lederach (1984, p.18) al entender que la educación para la paz es todo un proyecto no solo pedagógico sino también analítico, crítico y creativo. Educar para la paz es transmitir al individuo la autonomía suficiente para que pueda razonar y decidir con toda libertad. Es educar en el respeto a las normas cuando son justas y en la desobediencia cuando son injustas. Significa también proporcionarles criterios que les permitan defender sus diferencias sin violencia. Todos somos responsables de la educación para la paz. Sin lugar a dudas es un instrumento imprescindible para la transformación social y política.

Se podría decir que es un proceso en el que se adquieren valores y conocimientos, así como también actitudes, habilidades y comportamientos que son indispensables para alcanzar la paz, y que esta debe ser concebida para que todos podamos vivir en avenencia con nosotros mismos, con los demás y con el medioambiente. 


\section{VISIÓN DE LA PROBLEMÁTICA EN EL CARIBE COLOMBIANO}

Repensando el Caribe colombiano lo podríamos describir como una región donde conviven diversos tonos y matices, donde convergen múltiples culturas y se fusionan costumbres, festejos e idiosincrasia. Sin embargo, cabe apartarse de estos imaginarios para caracterizarla también como una región con un elevado índice de pobreza y la presencia de múltiples violencias que recorren todo el espectro de la clasificación de Galtung (1990, p.295): "directa, estructural, simbólica y cultural"; las cuales, en su conjunto tienen relación con la injusticia social. Estos elementos hacen parte de las relaciones arraigadas en los modelos violentos que asedian el sistema educativo no solo en la región Caribe sino también en toda Colombia.

No todas las expresiones de violencias son directas, sino que estas pueden ser invisibles y casi imperceptibles. Porque se encuentran de forma íntima, dentro de nuestro contexto social y también dentro de nuestras costumbres, patrones, modelos, imaginarios, saberes y actitudes que a veces se traspasan de padres a hijos. Se cree que estos comportamientos son normales, y no lo son. Por ese motivo es importante conocer los procesos educativos y verlos al mismo tiempo como un instrumento que modela al individuo, y que por lo tanto permea su personalidad, definen sus actitudes y comportamientos futuros.

En el Caribe, es innegable el rezago, la desigualdad y el atraso. Todas estas situaciones exponen de forma evidente la fragmentación del tejido social y este a su vez hace palpable un gran número de violencias que se ciernen alrededor del ámbito social y familiar afectando a todas aquellas personas que forman parte 
de la comunidad educativa como lo son: docentes, estudiantes, directivas, padres de familia, entre otros.

La situación de violencia por la que atraviesa el país, y por ende la región Caribe colombiana, hace evidente las inequidades sociales, (Galtung, 1998), pues no solo golpea a los ciudadanos de forma individual, sino también los lesiona en general. Tampoco la violencia puede ser atribuida a un solo individuo, a un grupo armado, a un grupo narcoterrorista o a una banda criminal, sino a todos los colombianos; estos deben comprometerse en alcanzar la paz, si desean un mejor país. Como ciudadanos responsables, debemos empezar a construir la paz todos los días desde lo cotidiano, en el hogar, en la academia, en la calle $y$, en general, en todos los espacios que adoptamos para desempeñarnos como ciudadanos y como individuos que forman parte de una sociedad en la cual se han destacado las dificultades, pero que sigue adelante a pesar de los nefastos y cruentos hechos que la afligen (Salamanca, 2008).

Hoy todos los colombianos deseamos un mejor país para nuestros hijos, y para lograrlo estamos luchando en la transformación de la sociedad desde lo individual, al entender que no podemos seguir esperando a que otros transformen nuestra realidad, sino que nosotros mismos debemos convertirnos en activos agentes sociales si anhelamos un mejor mañana. El reto es la suma de voluntades para alcanzar el sueño de la paz desde la casa o la oficina, en la profesión u oficio. Debemos aprender a respetar a los demás y rechazar todo aquello que no sea noble, digno y honesto. Se trata de concebir la paz no solamente como la ausencia del conflicto, sino también como la situación donde prevalezca la equidad y la justicia. 


\section{¿POR QUÉ DEBEMOS COMPROMETERNOS TODOS EN CONSTRUIR UNA CULTURA DE PAZ?}

Es una tarea con la que debemos comprometernos todas las personas porque de ello depende el futuro de la humanidad. Frente a este mundo globalizado plagado de violencia, tenemos una responsabilidad y un compromiso, que es el de transformar la escuela, la universidad y todos los centros educativos en fuerzas de paz. Instituciones como la UNESCO (1997) plantean que la educación superior es algo más que un simple nivel educativo, que en un período caracterizado por la cultura de guerra debería ser en nuestras sociedades la principal promotora al respecto dice: "de la solidaridad moral e intelectual de la humanidad y de una cultura de paz construida sobre la base de un desarrollo humano sostenible, inspirado en la justicia, la equidad, la libertad, la democracia y el respeto pleno de los derechos humanos" (39). Martínez (2010, p.62) concluye estas aspiraciones vinculándolas al buen gobierno, afirmando que "No hay paz sin justicia y buen gobierno, no hay buen gobierno sin paz y sin justicia, no hay justicia sin paz y buen gobierno". Es sin duda un modo de concretar aquella sentencia más general que nos legó Gandhi, en la que nos sugiere que no hay caminos para la paz, sino que la paz es el camino; y la violencia no es otra cosa que el miedo a los ideales ajenos.

Estos pensamientos y reflexiones nos llevan a repasar necesariamente que en la construcción de una cultura de paz es importante prevenir los conflictos, por ello todos debemos comprometernos a luchar utilizando como herramientas el diálogo y la negociación. Para que podamos cosechar los frutos de la cultura de paz debemos empezar a actuar desde ahora respetando, protegiendo y fomentando la libertad de expresión, de opinión, la 
igualdad entre hombres y mujeres, luchando contra toda forma de discriminación, viviendo en tolerancia y solidaridad.

La conquista de la paz es una tarea constante, que incumbe a toda la humanidad. Todos tenemos alguna responsabilidad por la violencia en la que vivimos, por el medio inicuo, indigno e inequitativo que hemos construido, por la indolencia y apatía con la que vemos la realidad, por querer sostener a nuestra sociedad con una doble cultura de legitimidad y de riqueza fácil, por hacer del conflicto y de la violencia un negocio. Por ello todos tenemos que comprometernos en la construcción de una cultura de paz para que podamos encontrar una salida. Todos debemos cultivar una pedagogía por la paz que nos conduzca mas allá de la tolerancia, aceptación y comprensión de las personas; y para ello necesitamos comprometernos no solo con la sociedad sino también con nuestras familias, construyendo procesos educativos serios que nos acompañen en la lucha contra este flagelo.

\section{EDUCACIÓN PARA LA PAZ Y PARA LA CONVIVENCIA EN COLOMBIA. INSTRUMENTOS NORMATIVOS}

En nuestro contexto, la Constitución política de 1991 le delegó a la educación responsabilidades con respecto a la formación para la paz y la convivencia, orientada a formar ciudadanos respetuosos de la diversidad, de la ley y las diferencias, con formación democrática y con la capacidad de negociar y de resolver sus conflictos.

La Ley 115 de 1994 establece que uno de los fines de la educación es la formación en el respeto a la vida y demás derechos humanos, a la paz, a los principios democráticos de convivencia, pluralismo, justicia, solidaridad y equidad, y también en el ejercicio de la tolerancia y la libertad. 
El Decreto 1860 de 1994 instaura pautas y objetivos para los manuales de convivencia escolar, los cuales deben incluir -entre otras normas de conducta- las que garanticen el mutuo respeto y procedimientos para resolver con oportunidad y justicia los conflictos.

La Ley 715 de 2001, que reglamenta los recursos y competencias para la prestación de servicios educativos y de salud, hace explicito que entre las competencias de la nación en materia de educación, está el formular las políticas y objetivos de desarrollo, para el sector educativo y dictar normas para la organización y prestación del servicio.

Por medio de la Ley 1732 de 2004 se establece la Cátedra de la Paz en todas las instituciones educativas del país; esta iniciativa va encaminada a generar la construcción de ambientes más pacíficos desde las aulas y es de obligatorio cumplimiento en todas las instituciones educativas del país.

El Decreto 1038 de 2015 reglamenta la Ley 1732 de esta cátedra, inquiriendo a "todas las instituciones educativas para que incluyan en sus planes de estudio la materia de Cátedra de La Paz antes del 31 de diciembre de 2015".

El plan decenal de educación 2006-2016 presenta el tema de la educación para la convivencia, la paz y la democracia, y propone como uno de los desafíos de la educación nacional el fortalecimiento de la sociedad civil y la promoción de la convivencia ciudadana.

La Republica de Colombia, a través del Ministerio de Educa- 
ción Nacional, con el propósito de dar aplicación a las leyes anteriormente mencionadas, ha elaborado a través de la política educativa unas orientaciones para la formación escolar en la convivencia. Esta ha venido adelantando acciones para responder a las necesidades educativas en temas como constitución, ética, democracia, valores, formación de identidad y convivencia. Además viene realizando esfuerzos para mejorar la calidad de la educación, ampliando la cobertura y contribuyendo, desde diferentes escenarios, a hacer de la escuela un espacio para la formación de procesos educativos que promuevan valores y desarrollen competencias individuales y de grupo para ejercer la democracia, interactuando desde el respeto a los derechos de los demás, manejando de manera adecuada los conflictos, con la participación de alternativas de solución a los problemas que afectan a la colectividad, de modo que se pueda alcanzar una sociedad más equitativa, justa y en paz.

Estas orientaciones pretenden fortalecer a las escuelas como instituciones forjadoras de sujetos competentes y capaces de establecer relaciones sociales y humanas dentro del marco del respeto y la tolerancia, concretando propuestas que conviertan los conflictos en una oportunidad para que puedan vivir juntos, adquiriendo además competencias para manejar adecuadamente los conflictos buscando soluciones a los problemas.

\section{PROPUESTA DE EDUCACIÓN Y CULTURA PARA LA PAZ}

El sistema escolar es un reflejo de la sociedad que en él se encuentra representada. Siguiendo a autores como Appel (1986, 1989, 1996), De Sousa (2007, 2011), Muñoz Cabrera (2011), Torres (2001), en su seno concurren tanto las tendencias a la reproducción de las estructuras y valores hegemónicos de aque- 
Ila, como los ideales de renovación, cambio o transformación, en el tema que nos ocupa, de la cultura de la violencia en cultura de paz. Por ello, el conflicto en la escolaridad, en la educación, no es muy diferente al conflicto y las tensiones que se dan en el contexto social más amplio. En los diferentes ámbitos y procesos escolares están presentes también las múltiples violencias que afectan a la población: la estructural, relacionada con la pobreza y la inequidad; pero también la cultural y simbólica, tanto en lo referido al desigual capital cultural de acceso y el modo en que el sistema refuerza su importancia durante la estancia en el mismo, como en lo que se refiere a la selección y legitimación de determinada cultura sobre otras posibles; así como de la opción por unas determinadas visiones, formas de pensar, sentir y vivir frente a otras. En sus diferentes componentes, la escuela y la escolaridad reproducen la violencia estructural e incuban la violencia simbólica y cultural en la producción de discursos y prácticas pedagógicas que, lejos de cuestionar aquella, la excusan, legitiman y refuerzan, contribuyendo a reproducir las condiciones y estructuras sociales vigentes. No es extraño entonces que haya consenso en considerar que la educación es un medio para construir sociedad, pero no siempre se explicita qué sociedad se desea construir y hacia dónde debería orientarse el proceso educativo o la formación de las generaciones más jóvenes. Analizando el caso colombiano, podemos identificar la decisiva influencia del modelo socioeconómico hegemónico, capitalismo financiero y mercado globalizado que se implementa y refuerza con políticas neoliberales; una tendencia radicalizada con la apertura de mercados en el Caribe.

El sistema escolar en Colombia sufrió cambios que en su gran mayoría provienen de la apertura de mercados. Por tanto es in- 
teresante conocer el pasado, en concreto la última década del siglo XX, en el que de manera paulatina la educación fue llevada a procesos que en su totalidad fueron nominados como revolución educativa, con el pretexto de estar en consonancia con los tiempos de la globalización (García Canclini, 1996). Este hecho histórico es fundamental, ya que sirve para comprender lo sucedido y ayuda en la construcción de una propuesta de intervención para la educación y la cultura de paz.

La Educación para la Paz se ha ido transformando en un nuevo desafío para la educación. Aunque su constitución e implantación en los centros escolares es todavía insuficiente, la Educación para la Paz no es una práctica circunstancial, es una necesidad, y por ello es importante realizar un trabajo de sensibilización a través de aquellas personas que son a su vez agentes sociales y que pueden transmitir estos valores al resto de la población.

Desde ese marco muy general pero con incidencia muy profunda, surge la inquietud para desarrollar una Propuesta de Educación y Cultura de Paz con cobertura a nivel regional. Su implementación ayudaría a tener centros educativos libres de violencias. Desde luego afectaría favorablemente al proceso de aprendizaje en niñas, niños, jóvenes, docentes y en toda la sociedad, ya que se empieza a promover una cultura de paz que se propicia y nace en el aula, como un ejercicio que le apostaría a una ciudadanía con perspectiva de paz; es decir, se estarían moldeando las sensibilidades, percepciones y actitudes de aprendices, que a futuro tendrían el interés de evidenciar sus competencias orientadas a la paciología; serian jóvenes con perfil de paciólogas y paciólogos, que afectarían a sus lugares más próximos, como la misma escuela, su hogar, el barrio, la iglesia que frecuentan o a su grupo de iguales. 
La propuesta de educación para la paz y la resolución de los conflictos que se expondrá a continuación pretende utilizar como herramienta de transformación social la fuerza de la educación-sensibilización, creando espacios para la reflexión y el sano razonamiento, para la propagación de prácticas de educación que ayuden a entender la responsabilidad que tienen todos frente al conflicto y la situación de violencia que existe alrededor de todos los colombianos.

Por ello se hace necesario educar desde una visión integradora y transformadora, arriesgando por una educación activa y participativa en donde consigamos fomentar un pensamiento dinámico, crítico y generador de ideas creativas. El vivir en paz es un valor que debemos aprender, es un esfuerzo que debemos hacer de forma conjunta y permanente.

Mayor Zaragoza (1994) afirma que se debe favorecer la razón, el diálogo y la conciliación (p.167), tres elementos fundamentales sin lugar a dudas para la interculturalidad, pues por medio de ellos se logra escuchar al otro, entendiéndolo y compartiendo con él lo que somos. La educación actual nos debe llevar a entrar en diálogo con la diversidad, la multiculturalidad y la pluralidad de pensamientos para entender esas diferencias que tanto separan al ser humano de sí mismo. Una cultura de paz, continúa diciendo, es necesaria para que lo que nazca en el espíritu del hombre sea totalmente contrario a lo que conocemos como guerra, y para ello debemos generar acciones, modos de vida, comportamientos, hábitos, actitudes que favorezcan y vayan a favor de la paz. En este sentido, Muñoz (2007) o Muñoz y Molina (2009) expresan que la idea de cultura de paz se sustenta en la necesidad de orientar e implementar un mundo más pacífico, pero también la 
consideran antídoto de la violencia y necesaria para gestionar la conflictividad. Otro ámbito en el que la cultura de paz tiene una importancia capital es el proyectivo. Necesitamos imaginar un futuro esperanzador, en el que la paz suponga un horizonte deseable por el cual esforzarse y que nos ayude a agentes, colectivos y organizaciones sociales e instituciones a superar el fatalismo.

En ese repensar la Educación y la cultura de paz para el Caribe colombiano podríamos tomar las ideas de autores como Banda (1998), Bastida (1994) o Grasa (Grasa, 1997) para quienes la Educación y la Cultura de Paz son dos conceptos íntimamente relacionados, que han venido adquiriendo una importancia especial en los estudios sobre la educación consciente para la paz, caracterizada por ayudar a construir valores y actitudes determinados por la justicia, la libertad, la cooperación, el respeto, la solidaridad, la actitud crítica, el compromiso, la autonomía, el diálogo y la participación. Al mismo tiempo se cuestionan los valores que son contrarios a la paz como la discriminación, la intolerancia, la violencia, el etnocentrismo, la indiferencia, el conformismo. Para Jares (1999, p.124) se trata de:

Un Proceso educativo dinámico, continuo y permanente, fundamentado en los conceptos de paz positiva y en la perspectiva creativa del conflicto como elementos significantes y definidores, que a través de la aplicación de enfoques socioafectivos y problematizantes pretende desarrollar un nuevo tipo de cultura, la cultura de la paz que ayude a las personas a desvelar críticamente la realidad para poder situarse ante ella y actuar en consecuencia.

El concepto de paz positiva (Galtung, 1993) hace hincapié precisamente en la necesidad de crear las condiciones de paz para 
prevenir la violencia. Educar en términos de paz positiva implica cuestionar todas las formas de violencia en las estructuras sociales, así como empeñarse en la búsqueda de la justicia social. Dicho de otro modo, significa analizar críticamente no solo la violencia que oprime, explota o mata, sino también la derivada de la autocracia política, de la explotación económica, de la discriminación y alienación social, cultural y política. De tal forma que, la vida bajo la pobreza, la enfermedad, la opresión, la explotación y el analfabetismo no son menos importantes (Galtung, 2003). Desde esta perspectiva educar para la paz supone recuperar la idea de paz positiva. Esto implica construir y potenciar en el proceso de aprendizaje unas relaciones fundamentadas en la paz entre alumnos, padres y profesores; entre el ciudadano y el poder. De ello se deriva la necesidad de afrontar los conflictos que se den en la vida de los centros educativos y en la sociedad de forma no violenta (Fernández Herrería, 2001).

Educar para la paz desde el currículo escolar implica contemplarlo en su dimensión transversal, de forma que afecte a los contenidos de todas las áreas o disciplinas que se estudian, pero también a la metodología y a la organización de los centros educativos, de modo que en la convivencia escolar cotidiana pueda experimentarse la participación democrática y el compromiso con la paz, afrontando los conflictos de forma dialógica y no violenta (Escudero, 1990). En la promoción de una cultura de paz es fundamental el compromiso social desde todas las esferas y que se generen políticas de intervención que lo refuercen. No se trata de educar para inhibir la iniciativa y el interés, sino para encauzar la actividad, el espíritu crítico y la creatividad en procesos valiosos para la comunidad, haciendo que todos nos sintamos partícipes en la construcción de la paz. 
Para profundizar en la cultura de paz se debe aprender a reconocer que todos somos generadores de conflictos en la escuela, en el hogar, en la universidad, en la oficina, en la sociedad, etc. $Y$ por lo tanto debemos responder de forma solidaria ante lo que sucede a nuestro alrededor, sintiéndonos ciudadanos afectados, responsables y comprometidos con nuestro entorno, desde el más próximo al más lejano. No debemos esperar a que el otro transforme su realidad individual para que este cambio pueda incidir en el contexto social en el que vivimos. Debemos entender que para salir de la problemática en la que vive nuestra región, nuestro país, debemos empezar por asumir el reto de transformarnos a nosotros mismos, ampliar nuestra mirada, pues nuestras formas de pensar, sentir, actuar y vivir inciden en uno $u$ otro sentido en los ámbitos y en los espacios en que nos desenvolvemos.

Como educadores necesitamos cuestionar nuestros propios posicionamientos profesionales, nuestras percepciones y actitudes hacia los estudiantes (García Vallinas, 1998, 2000). El reconocimiento del otro, de sus intereses y necesidades educativas y sociales, es un requisito indispensable para articular propuestas relevantes para su formación en términos de paz. La comunicación dialógica requiere de una actitud de acogida y de confianza para desarrollarse, que los estudiantes se perciban escuchados, valorados; que sientan que sus opiniones son tenidas en cuenta, que sus propuestas se integran en los proyectos de trabajo del aula. Urge desterrar, en este sentido, actitudes, expresiones y comportamientos docentes que ignoren, rebajen o anulen a los estudiantes como personas y como ciudadanos. Se requiere alentar el reconocimiento, la empatía, la tolerancia y la solidaridad en la cultura de los grupos y comunidades escolares, como 
ecosistemas que les permitan aprender y vivir experiencias de paz y no violencia que constituyan una referencia para sus vidas. Debemos entender que la paz es el camino y se construye todos los días desde lo cotidiano en dichos espacios. Debemos facilitar a nuestros niños y jóvenes herramientas para convivir en paz, estableciendo relaciones humanas superadoras de cualquier forma de violencia y activistas frente a la discriminación.

A continuación sugerimos algunos propósitos y estrategias para educar en la paz y desarrollar la cultura de paz en los centros educativos, de acuerdo a la conceptualización que hemos venido haciendo:

- Construir métodos de sensibilización, formación e intervención frente al conflicto entre los diversos actores, incluyendo a toda la comunidad educativa.

- Enseñar y aprender a afrontar los conflictos de forma no violenta, desarrollando estrategias que nos ayuden a resolver los problemas en el ámbito escolar; implementando la conciliación como alternativa de convivencia pacífica; creando centros de conciliación escolar que ofrezcan una salida a la problemática escolar en forma proactiva y pedagógica; promoviendo al interior de estos centros la costumbre de escuchar con seriedad, tratando de entender todos los mensajes, incluyendo aquellos con los que no estemos de acuerdo, buscando puntos de encuentro que nos ayuden a reducir las diferencias, posibilitando el entendimiento en situaciones conflictivas.

- Establecer grupos para el liderazgo de la convivencia y la cultura de la no-violencia, vinculándoles con experiencias significativas y estudios de caso sobre conflictos que se hayan presentado anteriormente en la comunidad, de modo que dicha reflexión fortalezca el compromiso con la con- 
vivencia pacífica y la recomposición del tejido social de la escuela.

- Aprender a construir de forma colectiva valores, comportamientos y habilidades sociales que nos identifiquen como personas y miembros de una comunidad; a alcanzar estrategias para potenciar el aprendizaje ciudadano, a través de una democracia cada vez más participativa e igualitaria, respondiendo solidariamente con las generaciones presentes y futuras.

- Capacitar a niños, jóvenes y adultos en procesos de democracia participativa, representativa y directa, así como en el conocimiento y apropiación de principios y valores cívicos.

- Sensibilizar a la comunidad educativa en la necesidad de reorientar los procesos de formación en derechos humanos y derecho internacional humanitario, así como la profundización y conocimiento de nuevos derechos sociales, económicos y culturales; pues construir una cultura de paz requiere como prioridad comprender el sentido y la necesidad de los mismos, así como su respeto efectivo y viabilidad para el desarrollo colectivo de dicha cultura de paz.

- Promover programas de investigación, sistematización, innovación y producción del saber pedagógico para la paz, la convivencia y la ciudadanía.

En este proceso formativo debemos incluir a las familias, porque un alumno habituado a la violencia al interior de su hogar, ya sea verbal, física, económica, etc. difícilmente podrá entenderla como tal, y tendrá la falsa creencia de que es un comportamiento normal. Pero justamente por eso, porque no podemos controlar lo que pasa dentro del seno familiar, los docentes nos hallamos ante la necesidad de ofrecer a nuestros alumnos las herramientas necesarias para que aprendan a analizar, comprender y rechazar 
la violencia en cualquier espacio de la sociedad, convirtiéndose este en el único camino que les asegurará una convivencia pacífica a lo largo de los años.

\section{CONCLUSIONES}

Todo lo expuesto anteriormente, permite precisar que la cultura de paz es una tentativa que ha sido contemplada a lo largo de la historia de la humanidad, que es un modo de organizar el mundo basado en el derecho sagrado de vivir juntos, que es un conjunto de valores, actitudes, tradiciones, comportamientos y estilos de vida que inspiran una forma constructiva y creativa de relacionarse para poder alcanzar, desde una visión holística, la armonía del ser humano consigo mismo, con los demás y con la naturaleza. Convivir en paz es un derecho humano reconocido a través del cuerpo jurídico colombiano, que es el que sostiene la democracia. La educación, desde el paradigma de la cultura de paz, representa un gran desafío para la humanidad; por ello se hace necesario considerar alternativas educativas que afronten los diferentes tipos de violencia a través del currículo de la educación básica, secundaria y superior con propuestas formativas y de contenido específicas de educación para la paz; así como de desarrollo de la cultura de paz en la propia estructura, organización, gestión y funcionamiento de los centros educativos.

\section{REFERENCIAS BIBLIOGRÁFICAS}

Appel, W. M. (1986). Ideología y currículo. Madrid: Akal. Appel, W. M. (1989). Maestros y textos. Una economía política de las relaciones de clase y de sexo en educación. Madrid: Paidós/MEC.

Appel, W. M. (1996). El conocimiento oficial. La educación demo-

crática en una era conservadora. Barcelona: Paidós.

Asamblea General de las Naciones Unidas. (1999). Declaración y 
programa de acción sobre una cultura de paz. AIRES/53/243. Recuperado de: http://www3.unesco.org/iycp/kits/sp_res243. pdf.

Banda, A. (1998). Educación para la paz. Enciclopedia General de la Educación, V-3. Buenos Aires: Océano.

Bastida, A. (1994). Desaprender la guerra. Una visión crítica de la educación para la paz. Barcelona: Icaria.

Congreso de Colombia (1 de septiembre de 2014). Cátedra de la paz. [ley 1732 de 2014]. DO: 49.261 recuperado de: http:// www.secretariasenado.gov.co/senado/basedoc/ley_1732_ 2014.html

Congreso de Colombia (21 de diciembre de 2001). Norma orgánica en materia de recursos. [Ley 715 de 2001]. DO: 44.654. Recuperado de: http://www.secretariasenado.gov.co/senado/ basedoc/ley_0715_2001.html

Congreso de Colombia (8 de febrero de 1994). Ley general de educación. [Ley 115 de 1994] DO:41.214. Recuperado de: http:// www.secretariasenado.gov.co/senado/basedoc/ley_0115_1994. html

De Sousa S. (2007). Conocer desde el sur. Para una cultura política emancipatoria. La Paz: CLASCO, CIDES-UMSA, Plural Editores.

De Sousa S, B. (2011). Para descolonizar occidente. Más allá del pensamiento abismal. Buenos Aires: CLASCO.

Escudero M, J. M. (1990). El desarrollo del curriculum y la educación para la paz. Revista de Pedagogía Social, (5), 4-51. Madrid: España.

Fenández, A. (1994). Educando para la Paz: nuevas propuestas. Trabajado recuperado de Seminario de Estudios sobre la paz y los conflictos. Universidad de Granada.

Fernández H, A. (2001). Educación y cultura de paz. En: López López, M. C.: Educar para la ciudadanía y la paz como proyecto intercultural. Granada: GEU, 9-20. 
Fisas, V. (2001). Educar para una cultura de paz. Recuperado de: http://www.blues.vab.es/incom/2004/cas/fisascas.htm/

Galtung, J. (1990). "Cultural violence". Journal of Peace Research, 27(3), 291-305.

Galtung, J. (1993). Los fundamentos de los estudios sobre la paz. En: Rubio, Ana. Presupuestos teóricos y éticos sobre la paz, Granada: Editorial Universidad de Granada, 1-45.

Galtung. J. (1998). Tras la violencia 3R: Reconstrucción, reconciliación, resolución. Afrontando los efectos visibles e invisibles de la guerra y la violencia. Bilbao: Bakeaz, Gemika Gogorakruz. Galtung, J. (2003). Paz por medios pacificas. Paz y conflicto, desarrollo y civilización. Bilbao: Bakeaz/Gemika Gogorakruz.

García C, N. (1996) Culturas en globalización. América Latina-Europa-Estados Unidos: libro de comercio e integración. Caracas, Nueva Sociedad y CLASCO.

García V, E. (1998). Como tela de araña. Racionalización del tiempo escolar y colonización del trabajo docente. Revista Tavira de Ciencias de la Educación, (15), 55-64.

García V, E. (2000). De cómo el profesorado de Educación Primaria percibe la diversidad cultural. Revista Tavira de Ciencias de la Educación, (17), 85-110.

Grasa, R. (1997). A vueltas con la paz y el desarrollo. Madrid: Catarata.

Harber, C. (1997). L'efficacité des écoles, l'education pour la democratie et la no violence. París: UNESCO, (ED-97/WS/23).

Jares, J. (1999). Educación para la paz. Su teoría y su práctica. Madrid: Popular.

Lederach, J. P. (1984). Educar para la paz. Barcelona: Fontarama. Mayor Z, F. (1994). La nueva página. Barcelona: Círculo de Lectores/UNESCO.

Ministerio de Educación Nacional. (3 de agosto de 1994). Reglamentación parcial de la Ley 115 de 1994. [Decreto 1860 de 
1994] DO: 41.473. Recuperado de: http://www.mineducacion. gov.co/1621/articles172061_archivo_pdf_decretoI860_94.pdf Ministerio de Educación Nacional (25 de mayo de 2015). Reglamentación de la Cátedra de la paz. [Decreto 1038 de2015]. DO: 49.522. Recuperado de: http://www.alcaldiabogota.gov. co/sisjur/normas/Normal.jsp?i=61735

Ministerio de Educación Nacional. (2016). Plan Nacional Decenal de Educación 2016-2026. Recuperado de: http://www.plandecenal.edu.co/cms/

Morachimo, L. y Piscoya L. (2004). Temas transversales y desarrollo sostenible. Lima: CIFO-DIEEPP.

Muñoz C. P. (2011). Violencias interseccionales. Debates feministas y marcos teóricos en el tema de pobreza y violencia contra las mujeres en Latinoamérica. Tegucigalpa: CA WN.

Muñoz, F. A. (2007). Cultura de paz para afrontar los conflictos, Instituto de Paz y Conflictos. España: Universidad de Granada. Muñoz, F. y Molina R. B. (2009). Una cultura de paz compleja y conflictiva. La búsqueda de equilibrios dinámicos. Instituto de Paz y Conflictos, Revista Paz y Conflictos. España: Universidad de Granada.

Martínez, C. (2010). Mujeres y Diosas mediadoras de paz, Género y Paz. Barcelona: Icaria.

Rodríguez R. M. (1994). Educar para la paz y la racionalidad comunicativa. En: Herrería Fernández, A.: Educando para la paz: Nuevas propuestas. Granada: Universidad de Granada.

Salamanca, E. (2008). Las prácticas de resolución de conflictos en América Latina. Bilbao: Universidad de Deusto.

Torres S. J. (2001). Educación en tiempos de neoliberalismo. Madrid: Morata.

UNESCO (1997). Hacia una nueva educación superior. Ediciones Cresalciunesco. Caracas, Venezuela. Recuperado de: http:// unesdoc.unesco.org/images/O014/001493/149330so.pdf 